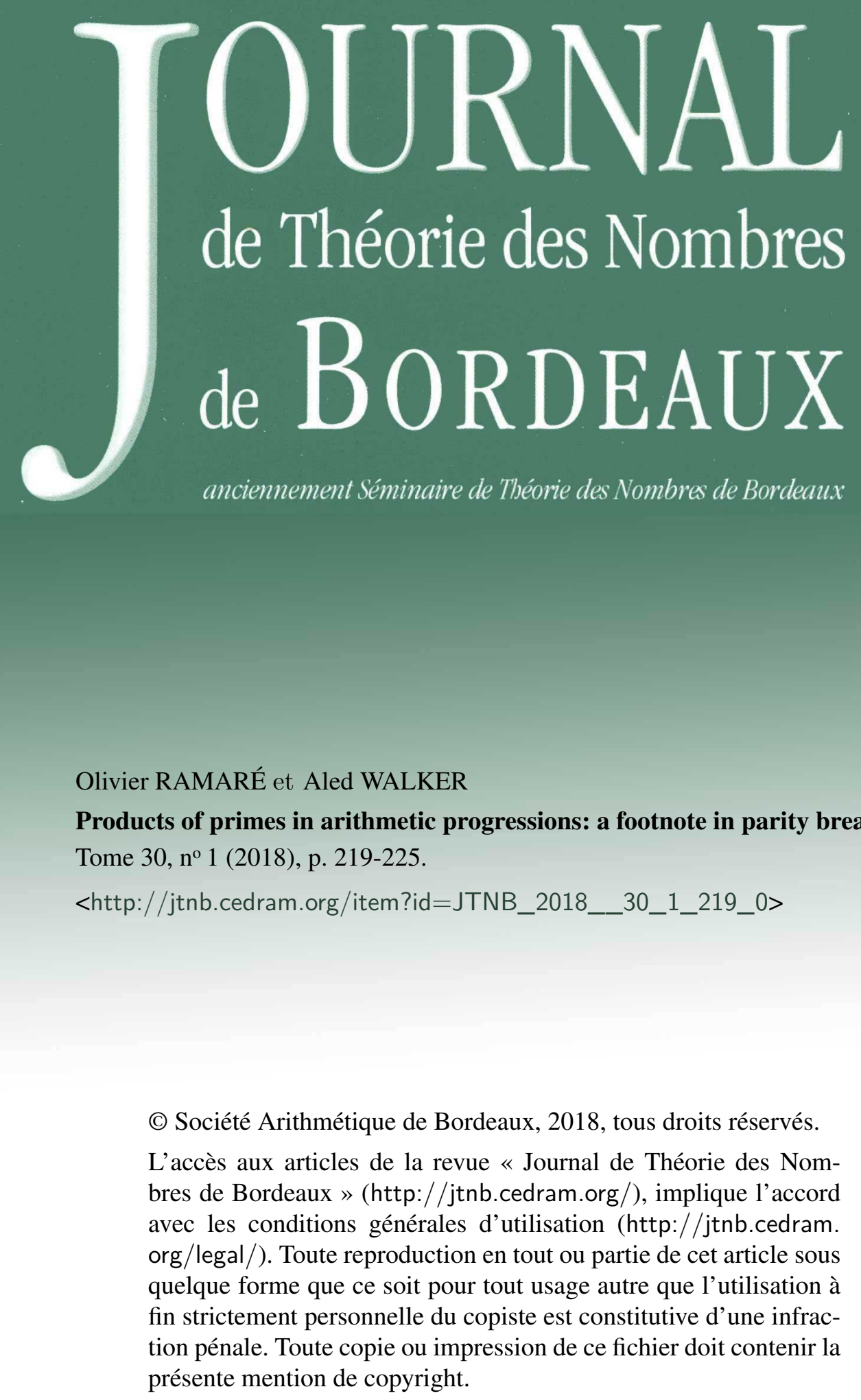

Olivier RAMARÉ et Aled WALKER

Products of primes in arithmetic progressions: a footnote in parity breaking

, no 1 (2018), p. 219-225.

(C) Société Arithmétique de Bordeaux, 2018, tous droits réservés.

L'accès aux articles de la revue « Journal de Théorie des Nombres de Bordeaux » (http://jtnb.cedram.org/), implique l'accord avec les conditions générales d'utilisation (http://jtnb.cedram. org/legal/). Toute reproduction en tout ou partie de cet article sous quelque forme que ce soit pour tout usage autre que l'utilisation à fin strictement personnelle du copiste est constitutive d'une infracpenale. Toute copie ou impression de ce fichier doit contenir la pesente mention de copyright.

\title{
cedram
}

Article mis en ligne dans le cadre du

Centre de diffusion des revues académiques de mathématiques http://www.cedram.org/ 


\title{
Products of primes in arithmetic progressions: a footnote in parity breaking
}

\author{
par OLIVIER RAMARÉ et ALED WALKER
}

\begin{abstract}
RÉSumÉ. Nous montrons que, étant donnés $x$ et $q \leqslant x^{1 / 16}$, toute classe inversible $a$ modulo $q$ contient au moins un produit d'exactement trois nombres premiers, chacun étant inférieur ou égal à $x^{1 / 3}$.

ABSTRACT. We prove that, if $x$ and $q \leqslant x^{1 / 16}$ are two parameters, then for any invertible residue class $a$ modulo $q$ there exists a product of exactly three primes, each one below $x^{1 / 3}$, that is congruent to a modulo $q$.
\end{abstract}

\section{Introduction and results}

Xylouris' version of Linnik's Theorem [15] tells us that, for every modulus $q$ and every invertible residue class $a$ modulo $q$, one can find a prime congruent to a modulo $q$ that is below $q^{5.18}$ provided $q$ be large enough. The proof relies on intricate techniques, and though the result is indeed effective, no one has been able to give any explicit version of it. The aim of this paper is to show that one can easily access a fully explicit result, with respectable constants, provided one replaces primes by products of three primes. Here is what we prove, by combining a simple sieve technique together with classical additive combinatorics.

Theorem 1.1. Let $x$ and $q \leq x^{1 / 16}$ be two parameters. Then for any invertible residue class a modulo $q$, there exists a product of three primes, all below $x^{1 / 3}$, that is congruent to a modulo $q$.

We did not try to be optimal in our treatment but sought the simplest argument. The main surprise is that we use sieve techniques in the form of Brun-Titchmarsh inequality but we are not blocked by the parity principle. The reader may argue that we use a lower bound for $L(1, \chi)$, but the bound we employ is the weakest possible and does not rely on Siegel's Theorem.

Manuscrit reçu le 22 mai 2016, révisé le 3 novembre 2016, accepté le 2 décembre 2016.

2010 Mathematics Subject Classification. 11N13, 11A41, 11N37, 11B13.

Mots-clefs. Primes in arithmetic progressions, Least prime quadratic residue, Linnik's Theorem.

The first author has been partly supported by the Indo-French Centre for the Promotion of Advanced Research - CEFIPRA, project No 5401-1. The second author was supported by the EPSRC Grant EP/M50659X/1. 
In particular, it is not strong enough to push a possible Siegel zero away from 1, a fact known to be equivalent to the parity phenomenom (see [8] and [11], or [12, Chapter 6] for a more complete discussion).

Our theorem is also linked with a conjecture of Erdös that says that every invertible congruence class should contain a product of two primes not more than $q$. This is discussed in work of the second author [14].

A numerically improved version is being prepared.

\section{Lemmas}

We begin with some crude bounds. Let us define

$$
f_{0}(q)=\prod_{p \mid q}(1-1 / \sqrt{p})^{-1} .
$$

Lemma 2.1. For $q \geqslant 2$ we have $f_{0}(q) \leq 3.32 \sqrt{q}$

Proof. For all primes $p$ we have $(1-1 / \sqrt{p})^{-1} \leqslant \alpha_{p} \sqrt{p}$, where

$$
\alpha_{p}= \begin{cases}\frac{1}{\sqrt{2}-1} & p=2 \\ \frac{1}{\sqrt{3}-1} & p=3 \\ 1 & \text { otherwise }\end{cases}
$$

and since $\alpha_{2} \leqslant 2.42$ and $\alpha_{3} \leqslant 1.37$, we obtain the inequalities

$$
f_{0}(q) \leqslant 2.42 \cdot 1.37 \cdot \sqrt{q} \leqslant 3.32 \cdot \sqrt{q} .
$$

We will also require a rudimentary estimate on $\phi(q)$.

Lemma 2.2. If $q \geqslant 31$ then $\phi(q)>8$.

Proof. Recall that

$$
\phi(n)=n \prod_{p \mid n}\left(1-\frac{1}{p}\right) .
$$

Therefore if $\phi(q) \leqslant 8$, the only prime factors of $q$ are $2,3,5,7$. By performing an easy case analysis on which of these primes divides $q$, one sees that the only $q$ for which $\phi(q) \leqslant 8$ are $1,2,3,4,5,6,7,8,9,10,12,15,20,24$, and 30 .

We will use the elementary theory of Dirichlet characters, referring the reader to the excellent monograph [2] of Davenport for an introduction on the subject. In particular we note the following easy bound.

Lemma 2.3. Let $\chi$ be a non-principal Dirichlet character modulo q. Let I be a subset of $\{1, \ldots, q\}$. We have

$$
\left|\sum_{n \in I} \chi(n)\right| \leq \phi(q) / 2
$$

The same bound holds true for any finite interval instead of I. 
Proof. We know on the one hand that, we have $\sum_{1 \leq n \leq q} \chi(n)=0$ by orthogonality, and on the other hand that $\chi(n)$ does not vanish only when $n$ belongs to the multiplicative group, say $\mathcal{U}_{q}$, of $\mathbb{Z} / q \mathbb{Z}$. We can hence bound $\left|\sum_{n \in I} \chi(n)\right|$ by the cardinal of $I \cap \mathcal{U}_{q}$ and by the cardinal of $\mathcal{U}_{q} \backslash I \cap \mathcal{U}_{q}$. One of them is not more than $\phi(q) / 2$, proving the first part of the lemma. When $I$ is a finite interval, we note that the sum of the values of $\chi(n)$ on any $q$ consecutive integers vanishes, reducing the problem to the first case.

We next modify an idea of Gel'fond from [4], which is maybe more easily read in $[5]$.

Lemma 2.4. Let $\chi$ be a non-principal quadratic character modulo q. We have

$$
L(1, \chi) \geq \frac{\pi}{4 \phi(q)}-\frac{\pi}{\phi(q)^{2}} .
$$

Proof. We consider the sum $S(\alpha)=\sum_{n \geq 1}(1 \star \chi)(n) e^{-n \alpha}$ for real positive $\alpha$. Since $(1 \star \chi)\left(m^{2}\right) \geq 1$ for every integer $m$, and $(1 \star \chi)(n) \geq 0$ in general, a comparison with an integral gives us

$$
1+S(\alpha) \geq \sum_{m \geq 0} e^{-m^{2} \alpha} \geq \int_{0}^{\infty} e^{-\alpha t^{2}} \mathrm{~d} t=\frac{\Gamma(1 / 2)}{2 \sqrt{\alpha}}=\frac{\sqrt{\pi}}{2 \sqrt{\alpha}} .
$$

On the other hand we can expand $(1 \star \chi)(n)=\sum_{d \mid n} \chi(d)$ and get

$$
S(\alpha)=\sum_{d \geq 1} \frac{\chi(d)}{e^{\alpha d}-1}=\frac{L(1, \chi)}{\alpha}-\sum_{d \geq 1} \chi(d) g(\alpha d)
$$

by using the non-negative non-increasing function $g(x)=\frac{1}{x}-\frac{1}{e^{x}-1}$. We find that, by Lemma 2.3,

$$
\begin{aligned}
\sum_{d \geq 1} \chi(d) g(\alpha d) & =-\sum_{d \geq 1} \chi(d) \int_{\alpha d}^{\infty} g^{\prime}(t) \mathrm{d} t \\
& =-\int_{0}^{\infty} \sum_{d \leq t / \alpha} \chi(d) g^{\prime}(t) \mathrm{d} t \\
& \geq \frac{\phi(q)}{2} \int_{0}^{\infty} g^{\prime}(t) \mathrm{d} t \\
& =-\phi(q) / 4
\end{aligned}
$$

since $\lim g(x)=1 / 2$ as $x$ tends to 0 from above. By comparing both upper and lower estimate for $S(\alpha)$, we reach

$$
L(1, \chi) \geq \frac{\sqrt{\pi \alpha}}{2}-\alpha-\frac{\alpha \phi(q)}{4} .
$$

We select $\alpha=\pi / \phi(q)^{2}$. The lemma follows. 
Lemma 2.5. Let $q \geq 3$ be an integer and $\chi$ be a non-principal quadratic character modulo $q$. Then there is a prime $p$ at most $q^{4}$ such that $\chi(p)=1$.

Proof. We adapt the proof of J. Pintz taken from [10]. Assume that no primes not more than a given real number $x$ are in the kernel of $\chi$. We use the notation $d \mid q^{\infty}$ to say that all the prime factors of $d$ divides $q$. Then on the one side we have

$$
\sum_{n \leq x}(1 \star \chi)(n)=\sum_{d \mid q^{\infty}} \sum_{\begin{array}{c}
m^{2} \leq x / d, \\
(m, q)=1
\end{array}} 1 \leq \sum_{d \mid q^{\infty}} \sqrt{\frac{x}{d}} \leq \sqrt{x} f_{0}(q)
$$

where $f_{0}$ is the function defined in (2.1), while on the other side we can approximate this sum by $L(1, \chi)$ as follows:

$$
\sum_{n \leq x}(1 \star \chi)(n)=\sum_{d \leq x} \chi(d)\left[\frac{x}{d}\right]=x \sum_{d \leq x} \frac{\chi(d)}{d}-\sum_{d \leq x} \chi(d)\left\{\frac{x}{d}\right\} .
$$

The first summation over $d$ is an approximation of $L(1, \chi)$ (recall Lemma 2.3):

$$
\begin{aligned}
L(1, \chi) & =\sum_{d \geq 1} \frac{\chi(d)}{d}=\sum_{d \leq x} \frac{\chi(d)}{d}+\int_{x}^{\infty} \sum_{x<d \leq t} \chi(d) \mathrm{d} t / t^{2} \\
& =\sum_{d \leq x} \frac{\chi(d)}{d}+\mathcal{O}^{*}\left(\frac{\phi(q)}{2 x}\right) .
\end{aligned}
$$

We treat the second summation in $d$ above by Axer's method from [1] (see also [7, Theorem 8.1]):

$$
\left|\sum_{d \leq x} \chi(d)\left\{\frac{x}{d}\right\}\right| \leq \sum_{d \leq y} 1+\sum_{m \leq x / y}\left|\sum_{d:[x / d]=m} \chi(d)\left\{\frac{x}{d}\right\}\right| \leq y+\frac{\phi(q) x}{2 y} \leq \sqrt{2 \phi(q) x}
$$

by selecting $y=\sqrt{\phi(q) x / 2}$, the second inequality following by Abel summation. All of this implies that $\sqrt{x} L(1, \chi) \leq f_{0}(q)+\sqrt{2 \phi(q)}+\phi(q) /(2 \sqrt{x})$. However, the previous lemma gives us a lower bound for $L(1, \chi)$ and thus we should have

$$
\frac{\pi}{4 \phi(q)}-\frac{\pi}{\phi(q)^{2}} \leq \frac{f_{0}(q)}{\sqrt{x}}+\sqrt{\frac{2 \phi(q)}{x}}+\frac{\phi(q)}{2 x} .
$$

We substitute $x=q^{4}$, using the upper bound for $f_{0}(q)$ provided by Lemma 2.1. Replacing the left hand side of the above inequality by $\pi / 8 \phi(q)$, which is permissible by Lemma 2.2 , together with the bound $\phi(q) \leq q$, after a short calculation we derive a contradiction for all $q \geq 45$. Calculating using the exact expressions for $f_{0}(q)$ and $\phi(q)$ when $q \in\{15, \ldots, 45\}$, we also derive a contraction. For the remaining $q$ it is easy enough to find 
primes $p \leqslant q^{4}$ such that $p \equiv 1$ modulo $q$. Indeed, for $q=2, \ldots, 14$ we may take $p=3,7,5,11,7,29,17,19,11,23,37,53,29$ respectively.

We quote the following result from [6], which is a strong form of the Brun-Titchmarsh inequality.

Lemma 2.6. When $1 \leq q<x$, we have

$$
\sum_{\substack{y<p \leq y+x, p \equiv a[q]}} 1 \leq \frac{2 x}{\phi(q) \log (x / q)} .
$$

for any positive $y$.

Lemma 2.7. We have $\pi(x) \geq x /(\log x-1)$ when $x \geq 5393$. Furthermore the number of primes not more than $x$ but prime to some fixed modulus $q$ below $x$ is at least $x / \log x$, again when $x \geq 5393$.

Proof. The first inequality is taken from [3]. For the second, we simply note that the number of prime factors of $q$ is at most $(\log x) / \log 2$ and that

$$
\frac{x}{\log x-1}-\frac{\log x}{\log 2} \geq \frac{x}{\log x}
$$

when $x \geq 5000$.

The final ingredient in the argument will be Kneser's Theorem, which we now recall (see [9, Theorem 4.3] or [13, Theorem 5.5]).

Lemma 2.8. Let $A$ and $B$ be two subsets of the finite abelian group $G$. Let $H$ be the subgroup of elements $h$ of $G$ that stabilizes $A+B$, i.e. that are such that $h+A+B=A+B$. We have

$$
|A+B| \geq|A+H|+|B+H|-|H| .
$$

\section{Proof of Theorem 1.1}

Let us first treat the case $x \geq 10^{16}$.

Let $X=x^{1 / 3}$. Since this parameter is at least $10^{5}$, Lemma 2.7 tells us that the number $\pi_{q}(X)$ of primes below $X$ which are coprime to $q$ is at least $X / \log X$. The Brun-Titchmarsh inequality in the form given by Montgomery \& Vaughan, recalled in Lemma 2.6, tells us that the number of primes less than $X$ in any progression $a \bmod q$, for $a$ prime to $q$, is at most $\frac{32}{13} X /(\phi(q) \log X)$. This implies, when compared to the total number of primes coprime to $q$ given by Lemma 2.7 , that at least $\frac{13}{32} \phi(q)$ such residue classes contain a prime. Let us call this set of classes $\mathscr{A}$ and apply Kneser's Theorem (Lemma 2.8) to the group $G$ of invertible residues modulo $q$. Let $H$ be the stabilizer of $\mathscr{A} \cdot \mathscr{A}$. We divide into cases according to the index of $H$. 
If $H$ is equal to $G$ then, since $\mathscr{A} \cdot \mathscr{A} \cdot H=\mathscr{A} \cdot \mathscr{A}$, we have $\mathscr{A} \cdot \mathscr{A}=G$ and of course $\mathscr{A} \cdot \mathscr{A} \cdot \mathscr{A}=G$.

If $H$ has index 2, then it is the kernel of some quadratic character $\chi$. Because $\mathscr{A}$ generates $G$ multiplicatively, there is a point $a$ in $\mathscr{A}$ such that $\chi(a)=-1$. By Lemma 2.5, there is another one, say $a^{\prime}$, such that $\chi\left(a^{\prime}\right)=1$. Hence $\mathscr{A} \cdot \mathscr{A}$ also has a point $b$ such that $\chi(b)=1$ and one, say $b^{\prime}$, such that $\chi\left(b^{\prime}\right)=-1$. This implies that $\mathscr{A} \cdot \mathscr{A} \cdot H=G$, i.e. $\mathscr{A} \cdot \mathscr{A}=G$.

When $H$ is of index 3 , then $\mathscr{A} \cdot H$ covers at least $2 H$-cosets (since $\frac{13}{32}>\frac{1}{3}$ ) and is thus of cardinality at least $2 \phi(q) / 3$. Kneser's Theorem ensures that $|\mathscr{A} \cdot \mathscr{A}| \geq \phi(q)$, i.e. that again $\mathscr{A} \cdot \mathscr{A}=G$.

When $H$ is of index 4 , then $\mathscr{A} \cdot H$ covers at least $2 H$-cosets (since $\left.\frac{13}{32}>\frac{1}{4}\right)$ and is thus of cardinality at least $\phi(q) / 2$. By Kneser's Theorem,

$$
|\mathscr{A} \cdot \mathscr{A}| \geq 2|\mathscr{A} \cdot H|-|H| \geq \frac{3}{4} \phi(q)
$$

When $H$ is of index $Y$ say, with $Y$ at least 5 , let us write $|\mathscr{A}| / \phi(q)=$ $1 / U$. The set $\mathscr{A} \cdot H$ is made out of at least $\lceil Y / U\rceil$ cosets modulo $H$. Using the same manipulation as above, Kneser's Theorem ensures that $|\mathscr{A} \cdot \mathscr{A}| / \phi(q) \geq(2\lceil Y / U\rceil-1) / Y$. A quick computation shows that the minimum of $(2\lceil Y / U\rceil-1) / Y$ when $Y$ ranges $\{5,6,7,8,9\}$ is reached at $Y=7$ and has value $5 / 7$. When $Y$ is larger than 10 , we directly check that $(2\lceil Y / U\rceil-1) / Y \geq \frac{2}{U}-\frac{1}{Y} \geq \frac{13}{16}-\frac{1}{10} \geq \frac{7}{10}$.

Combining these final two cases, we have proved that $|\mathscr{A} \cdot \mathscr{A}| \geq \frac{7}{10} \phi(q)$. Let $b$ be an arbitrary invertible residue class modulo $q$. The set $b / \mathscr{A}$ is of cardinality at least $|\mathscr{A}|$ and, since $\frac{13}{32}$ is greater then $\frac{3}{10}$, this is strictly larger than the size of the complementary set of $\mathscr{A} \cdot \mathscr{A}$. Therefore these sets have a point in common: there exist $a, a_{1}$ and $a_{2}$, all three in $\mathscr{A}$ such that $b / a=a_{1} a_{2}$, proving our theorem in this case.

It remains to deal with $x<10^{16}$, which is done by explicit calculation. The inclusion of this addendum was kindly suggested to us by an anonymous referee. Indeed, when $x<10^{16}$, the modulus $q$ is restricted to be not more than 10, implying that only a limited number of congruence classes are to be looked at. We proceed by hand:

- when $q=2$, we only need $x \geq 3^{3}$;

- when $q=3$, we only need $x \geq 7^{3}$;

- when $q=4$, we only need $x \geq 5^{3}$;

- when $q=5$, we only need $x \geq 19^{3}$;

- when $q=6$, we only need $x \geq 11^{3}$;

- when $q=7$, we only need $x \geq 29^{3}$;

- when $q=8$, we only need $x \geq 23^{3}$;

- when $q=9$, we only need $x \geq 23^{3}$;

- when $q=10$, we only need $x \geq 19^{3}$. 
This takes care of the situation when $x \geq 29^{3}$. However, when $x$ is below $29^{3}$, the bound $x^{1 / 16}$ is less than 2 . This ends the proof of our theorem.

\section{References}

[1] A. Axer, "Über einige Grenzwertsätze", Wien. Ber. 120 (1911), p. 1253-1298.

[2] H. Davenport, Multiplicative number theory, 3rd ed., Graduate Texts in Mathematics, vol. 74, Springer, 2000, $\mathrm{x}+177$ pages.

[3] P. Dusart, "Estimates of some functions over primes without R.H.", https://arxiv.org/ abs/1002.0442, 2010.

[4] A. O. Gel'Fond, "On the arithmetic equivalent of analyticity of the Dirichlet $L$-series on the line Re $s=1$ ", Izv. Akad. Nauk SSSR. Ser. Mat. 20 (1956), p. 145-166.

[5] A. O. Gel'FOND \& Y. V. LinNiK, Elementary methods in analytic number theory, Rand McNally \& Co., 1965, Translated by Amiel Feinstein. Revised and edited by L. J. Mordell, 242 pages.

[6] H. L. Montgomery \& R. C. Vaughan, "Hilbert's inequality", J. Lond. Math. Soc. 8 (1974), p. $73-82$.

[7] - Multiplicative Number Theory: I. Classical Theory, Cambridge Studies in Advanced Mathematics, vol. 97, Cambridge University Press, 2007, xvii+552 pages.

[8] Y. Мотонаshi, "A note on Siegel's zeros", Proc. Japan Acad., Ser. A 55 (1979), p. 190-192.

[9] M. B. Nathanson, Additive Number Theory -Inverse Problems and the Geometry of Sumsets, Graduate Texts in Mathematics, vol. 165, Springer, 1996, xiv+293 pages.

[10] J. Pintz, "Elementary methods in the theory of $L$-functions, VI. On the least prime quadratic residue $(\bmod \rho)$ ", Acta Arith. 32 (1977), p. 173-178.

[11] K. Ramachandra, A. Sankaranarayanan \& K. Srinivas, "Ramanujan's lattice point problem, prime number theory and other remarks", Hardy-Ramanujan J. 19 (1996), p. 256.

[12] O. Ramaré, Arithmetical aspects of the large sieve inequality, Harish-Chandra Research Institute Lecture Notes, vol. 1, Hindustan Book Agency, 2009, x+201 pages.

[13] T. TAo \& V. H. Vu, Additive Combinatorics, Cambridge Studies in Advanced Mathematics, vol. 105, Cambridge University Press, 2006, xviii+512 pages.

[14] A. Walker, "A multiplicative analogue of Schnirelmann's theorem", Bull. Lond. Math. Soc. 48 (2016), no. 6, p. 1018-1028.

[15] T. XYlOuRIS, "On the least prime in an arithmetic progression and estimates for the zeros of Dirichlet L-functions", Acta Arith. 150 (2011), no. 1, p. 65-91.

Olivier RAMARÉ

CNRS / Institut de Mathématiques de Marseille

Aix Marseille Université, U.M.R. 7373

Site Sud, Campus de Luminy, Case 907

13288 MARSEILLE Cedex 9, France

E-mail: olivier.ramare@univ-amu.fr

$U R L$ : http://iml.univ-mrs.fr/ ramare/

Aled WALKER

Mathematical Institute

University of Oxford

Andrew Wiles Building

Radcliffe Observatory Quarter

Woodstock Road

Oxford

OX2 6GG, United Kingdom

E-mail: walker@maths.ox.ac.uk

URL: https://www.maths.ox.ac.uk/people/aled.walker 